\title{
Hubble Space Telescope Images of Nuclear Rings in Barred Galaxies
}

\author{
Aaron J. Barth, Luis C. Ho, Alexei V. Filippenko \\ Department of Astronomy, University of California, Berkeley CA 94720 \\ Varoujan Gorjian, Matthew A. Malkan \\ Department of Astronomy, UCLA, Los Angeles, CA 90024 \\ Wallace L. W. Sargent \\ Palomar Observatory, Caltech, Pasadena, CA 91125
}

\begin{abstract}
We present new and archival Hubble Space Telescope (HST) images of circumnuclear star-forming rings in barred spiral galaxies. We find that nuclear rings in barred galaxies are composed of large numbers of super star clusters similar to those found recently in other types of starburst systems. In NGC 1097 and NGC 6951, the young clusters have absolute magnitudes of up to $M_{V}=-14$ or -15 , depending on highly uncertain extinction corrections, and effective radii of $2-3 \mathrm{pc}$. The images (especially that of NGC 6951) also show intricate spiral dust lane structure interior to the rings.
\end{abstract}

\section{Introduction}

Many barred spirals contain bright "hot-spots" of star formation near their nuclei, often arranged in a ring or a spiral pattern. Such structures are well understood to result from bar-driven inflow of gas to an inner Lindblad resonance (e.g., Tubbs 1982; Combes \& Gerin 1985; Athanassoula 1992). Many nuclear ring galaxies are known (Buta \& Crocker 1993) and several have been studied in detail (e.g., Hummel et al. 1987), but because of their small angular sizes, it is very difficult to resolve structure in the rings from the ground. The high spatial resolution of $H S T$ allows us to examine the detailed structure of the rings for the first time.

\section{Observations and Discussion}

We have compiled a collection of several new and archival $H S T$ images of nuclear rings observed with the WFPC and WFPC2 cameras. The observations were obtained through a variety of filters, including F547M, F555W, and F606W. The images displayed in Figures 1 and 2 have been rotated so that north is up and east is to the left. Other images not shown here include NGC 7469, IRAS F12495-1308, Mrk 42, Mrk 493, and Mrk 896. 

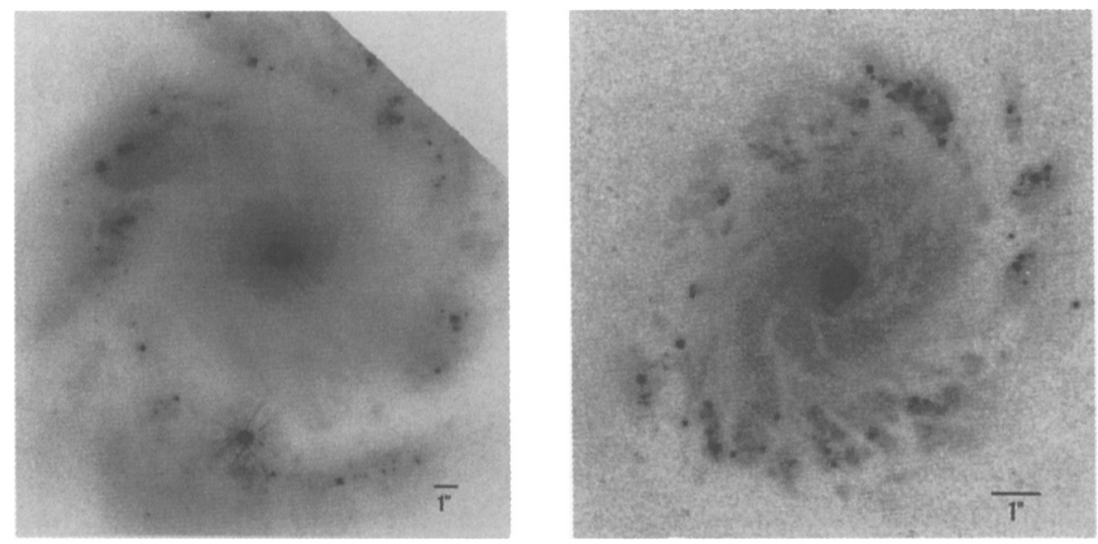

Figure 1. Nuclear rings in NGC 1097 (WFPC1/PC, F555W) and NGC 6951 (WFPC2/PC, F547M). The bright object south of the nucleus of NGC 1097 is SN 1992bd.

The nuclear rings are typically $\sim 1.5 \mathrm{kpc}$ in diameter, and show a wide variety of morphologies. In general, the more strongly barred galaxies appear to have the most regular, symmetric rings. Most of the rings contain bright, compact star clusters. We can assign ages of $\leq 10^{7} \mathrm{yr}$ to at least some of these clusters because many of the rings are known to be bright $\mathrm{H} \alpha$ emitters. In most cases, the star clusters occur only in the rings, and not in the regions interior to the rings.

We have measured the magnitudes and effective radii of the clusters that appear in the nuclear rings of NGC 1097 and NGC 6951 (Barth et al. 1995). These nuclear rings are very heavily obscured, with the Balmer line ratios indicating typical extinctions of $A_{V}=1.1 \mathrm{mag}$ and $3.4 \mathrm{mag}$, respectively, in the two galaxies. Using these highly uncertain numbers to correct the magnitudes, the absolute magnitudes for some clusters are as bright as $\mathrm{M}_{V}=-14$ to -15 . The clusters have effective radii of 1.3 to $4.0 \mathrm{pc}$ in NGC 1097 and $\leq 4 \mathrm{pc}$ in NGC 6951. The combination of such high luminosities and small radii indicates that these objects are "super star clusters" similar to those found recently in other types of starburst systems such as merger remnants and amorphous galaxies (e.g., Holtzman et al. 1992; O'Connell et al. 1994; Whitmore \& Schweizer 1995; O'Connell et al. 1995). These other systems are peculiar and strongly disturbed; the nuclear rings are the only known environment in which large numbers of super star clusters are found in normal galaxies. These clusters seem to be present-day analogs of young globular clusters, although their location in galactic disks suggests that they may disrupt at a young age, or spiral in to the nuclei due to dynamical friction (Tremaine et al. 1975), rather than remain as bound globulars.

The spiral dust lane structure seen interior to some of the rings (especially NGC 6951) suggests a possible connection between the large-scale gas flows and the fueling of active nuclei. (NGC 6951 and NGC 1019 are Seyfert galaxies, while NGC 1097 is a LINER.) However, it is not clear whether the gas in these 

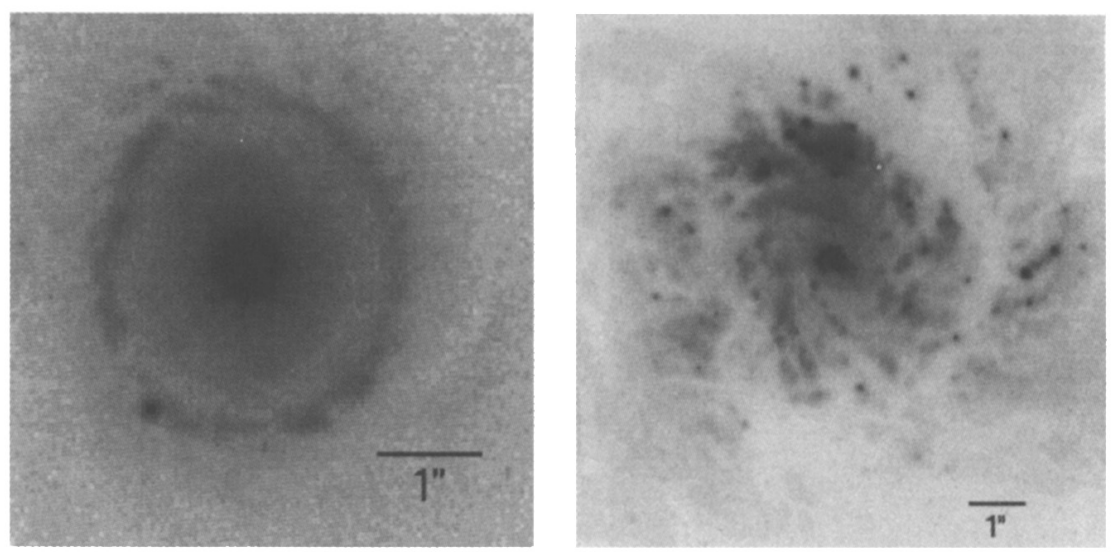

Figure 2. Nuclear rings in NGC 1019 (WFPC2/PC, F606W) and NGC 7552 (WFPC2/PC, F606W).

regions is inflowing or outflowing, and a spiral dust lane pattern inside a nuclear ring may in fact be representative of outflow (Combes 1988). Some simulations predict long periods of relative quiescence punctuated by rapid bursts of inflow to the nucleus (Wada \& Habe 1992; Heller \& Shlosman 1994).

Support for this work was provided by NASA through grants AR-4911.0192A, GO-5419.02-93A, and GO-5479.01 from STScI.

\section{References}

Athanassoula, E. 1992, MNRAS, 259, 345

Barth, A. J., Ho, L. C., Filippenko, A. V., \& Sargent, W. L. W. 1995, AJ, 110, 1009

Buta, R. \& Crocker, D. A. 1993, AJ, 105, 1344

Combes, F. 1988, in Galactic and Extragalactic Star Formation, R. E. Pudritz \& M. Fich, Dordrecht: Kluwer, 475

Combes, F. \& Gerin, M. 1985, A\&A, 150, 327

Heller, C. H. \& Shlosman, I. 1994, ApJ, 424, 84

Holtzman, J. A., et al. 1992, AJ, 103, 691

Hummel, E., van der Hulst, J. M., \& Keel, W. C. 1987, A\&A, 172, 32

O'Connell, R. W., Gallagher, J. S., \& Hunter, D. A. 1994, ApJ, 433, 65

O'Connell, R. W., Gallagher, J. S., Hunter, D. A., \& Colley, W. N. 1995, ApJ, 446, L1

Tremaine, S. D., Ostriker, J. P., \& Spitzer, L. 1975, ApJ, 196, 407

Tubbs, A. D. 1982, ApJ, 255, 458

Wada, K. \& Habe, A. 1992, MNRAS, 258, 82

Whitmore, B. C. \& Schweizer, F. 1995, AJ, 109, 960 